\title{
PERSPECTIVES POUR LA RECHERCHE SUR LES DETERMINANTS DE L'OFFRE D'INFORMATION DES ENTREPRISES.
}

\author{
Céline Mıchä̈LESCo
}

Résumé :

La recherche sur les déterminants de l'offre d'information des entreprises souffre de nombreuses limites inhérentes aux méthodes quantitatives utilisées qui répondent à une problématique positiviste. La validation statistique traditionnelle des déterminants simplifie le processus de communication sous-jacent et sa dynamique. S'attacher à l'étude de ce processus en adoptant une approche intégrée offre l'opportunité d'aborder d'autres questionnements concernant la communication comptable. Ce qui ouvre à de nouvelles démarches méthodologiques riches de perspectives.

Mots clefs: diffusion d'informations - variables explicatives - processus de communication - méthodologies d'investigation.

\section{Abstract:}

Current research about determinants of accounting information disclosed by companies suffers from various limits liked with quantitative tools suggested by positivist methodologies. Statistical validation of disclosure determinants hides the firm communication process and its dynamic. To investigate this process through an integrated, holistic approach suggests new interesting methodologies and problematics.

Key words : disclosure determinants - communication process model - quantitative and qualitative methodologies.

Correspondance : IUT de Paris

143 avenue de Versailles

75116 PARIS

celine.michailesco@iut.univ-paris5.fr 


\section{Introduction.}

Les études quantitatives s'intéressant aux déterminants de l'offre d'information des entreprises constituent depuis les années soixante un thème de recherche prolifique. L'objectif est de prouver l'incidence de quelques variables caractéristiques de l'entreprise (taille, diffusion du capital, structure financière, statut de cotation, etc.) sur la qualité de l'information (ou plus exactement la quantité d'informations) qu'elle diffuse, évaluée par le moyen d'un indicateur (une liste d'item associée à un système de pondération). L'intérêt des résultats apportés pour l'orientation de l'action des commissions d'opérations de bourse ou du normalisateur, a largement contribué à leur réalisation dans le cadre des pays en voie de développement ou des pays européens comme la France (Michaïlesco, 1999).

Théorie de l'agence et théorie positive confortent traditionnellement la légitimité de ce type de recherche en privilégiant les déterminants du processus de communication au détriment de l'analyse du processus en lui-même. Or, une telle approche quantitative de la diffusion d'information et de ses déterminants, est simplificatrice voire réductrice sous trois aspects. En premier lieu, l'aspect décisionnel de la diffusion d'information est occulté par la méthodologie d'évaluation de l'offre d'information. L'indicateur de diffusion, et l'indice qu'il permet de calculer, "canalise » en une valeur numérique un processus de communication complexe fait de jugements, d'arbitrages de décisions tenant compte de multiples contraintes de l'environnement externe et interne à l'entreprise. L'indice de diffusion peut être considéré comme une proxy du processus de communication et des décisions qui modèlent l'offre d'information. Ainsi, les tests statistiques révèlent les facteurs qui ont le plus pesé sur le processus en mettent en évidence des corrélations entre les variables explicatives et l'offre d'information. Ils confirment ou infirment ainsi la prise en compte des facteurs considérés au cours du processus de communication. Mais ils n'apportent aucune information sur la manière dont les variables étudiées affectent le processus : la nature des influences exercées est occultée. Ceci est renforcé par un dernier aspect: les variables opérationnelles adoptées concourent, elles aussi, à la simplification des mécanismes par lesquels certains facteurs affectent le processus de communication.

Si la simplification du processus de communication, tant dans les facteurs considérés que dans le recours à un indice de diffusion, est nécessaire dans le cadre d'une étude quantitative et contribue à éclairer le phénomène étudié, elle masque, d'une part, que l'ensemble des facteurs susceptibles d'influencer l'offre d'information d'une entreprise est vaste: plus vaste que les seuls facteurs opérationalisables quantitativement. D'autre part, de par cette simplification, aucune information n'est apportée sur les caractéristiques des relations qui s'établissent entre les facteurs étudiés et la qualité de la diffusion. Ainsi, une approche intégrée consistant en une modélisation $\mathrm{du}$ processus de communication offre l'opportunité de le reconstituer dans sa complexité, de préciser quels facteurs peuvent être pris en considération et de quelle manière ils agissent sur le processus et interagissent entre eux. Cette proposition invite ainsi à reconsidérer les démarches traditionnelles de validation quantitative, tant dans le choix des relations testées que dans la mise en œuvre de ces tests, et à se focaliser désormais sur la manière dont ces déterminants sont pris en considération et agissent au sein des organisations. Elle montre également l'intérêt des méthodologies d'investigation qualitative de l'offre d'information comptable et de ses déterminants et les pistes de recherche ouvertes. 
Notre réflexion sur les enjeux de la recherche sur les déterminants de l'offre d'information comptable par une entreprise est présentée en deux temps. Nous exposons dans une première partie une revue des éléments (étapes et facteurs explicatifs) constituant le processus de communication de l'information comptable. Dans une seconde partie, nous proposons une analyse de ce processus axée sur les relations qui unissent les facteurs entre eux et à cette offre d'information externe. Ceci nous amène à une classification de chacune des relations identifiées et à une modélisation du processus de communication. Nous revenons en conclusion sur l'intérêt de cette réflexion pour la recherche à venir sur l'offre d'information des entreprises.

\section{L'apport de la littérature à la reconstitution du processus de communication d'information comptable.}

Reconstituer le processus de communication permet de faire ressortir la dimension volontariste de la diffusion d'information dont la fonction est de rendre l'entreprise visible à son environnement en véhiculant des données particulières susceptibles de satisfaire différentes parties prenantes et de servir les objectifs économiques et sociaux de l'entreprise. L'information comptable résulte donc de choix stratégiques visant à conforter la marge d'action et de négociation de l'entreprise et à assurer la réalisation de ses objectifs (Hopwood, 1983). En conséquence, ces choix tiennent compte des pressions exercées par les partenaires, perçues et interprétées, d'objectifs particuliers et des structures internes.

La littérature tant théorique qu'empirique considère que le processus de communication se déroule en quatre étapes : une étape de formalisation de la stratégie de communication, au cours de laquelle sont définis des objectifs en fonction des pressions externes, une étape de mise en œuvre de la stratégie, c'est-à-dire une étape de production de l'information, contrainte par les structures internes et une étape de communication externe proprement dite de l'information. Enfin, le processus intègre une dernière étape qui est celle de l'apprentissage généré par le retour sur communication. Cette étape vise à comparer les résultats obtenus par rapport aux objectifs poursuivis par la stratégie. A chacune de ces étapes interviennent différents facteurs qui influencent le processus de communication et s'influencent mutuellement et que nous allons passer en revue.

\subsection{L'étape de l'élaboration de la stratégie de communication.}

La stratégie de communication est l'interface fondamentale qui explique l'offre d'informations externes d'une entreprise. Cette stratégie possède deux fonctions (Crozier et Friedberg, 1977) : contraindre l'environnement afin d'atteindre les objectifs économiques qu'elle poursuit (stratégie offensive) et échapper à sa contrainte en protégeant ses marges d'actions (stratégie défensive). L'objet d'une stratégie est avant tout, pour l'entreprise, celui de réduire des coûts de diverses natures.

\subsubsection{La stratégie de communication.}

La stratégie peut être définie comme la base schématique de l'élaboration des documents externes. Elle est le résultat de multiples arbitrages : 
- Un arbitrage sur le contenu de la diffusion en fonction des différents coûts directs et indirects impliqués (coûts de la diffusion, coût de propriété, coûts politiques, etc.) et des bénéfices potentiels en terme de coût du capital, d'image de l'entreprise ;

- Un arbitrage entre les différentes sources de financement accessibles à l'entreprise d'après les obligations formelles ou informelles de diffusion qui leurs sont associées. Certains dirigeants préféreront partager l'information de manière confidentielle avec les propriétaires du capital ou des banquiers plutôt qu'avec des investisseurs (Healy et Palepu, 1993) ;

- Un arbitrage entre diffusion et signaux substitutifs ou complémentaires (Healy et Palepu, 1993). Les dirigeants peuvent contrebalancer une communication de médiocre qualité (ce qui pourrait nuire au cours des actions) par une politique de distribution de dividendes en augmentation. Cet arbitrage peut également résulter de la régulation comptable en vigueur. Informations volontaires et informations obligatoires n'étant pas toujours complémentaires (Dye, 1985), une augmentation des informations obligatoires peut réduire les informations volontairement diffusées auparavant, les entreprises cherchant à conserver privées certaines informations.

La base schématique résulte également des objectifs, variables, poursuivis à travers la diffusion d'information : aider à la gestion de l'entreprise, permettre aux investisseurs de prendre les décisions adéquates, corriger des mauvaises évaluations, modifier l'actionnariat, rechercher d'avantages compétitifs. Elle se traduit dans le contenu de la communication qui prend la forme d'une simple application des principes comptables généralement admis (niveau élémentaire), d'une diffusion volontaire sur le long terme, ou enfin de l'envoi de signaux variés pour renforcer le contenu informationnel des rapports annuels (accroissement des parts du capital détenues par les dirigeants, accroissement du ratio d'endettement, diffusion de rapports d'experts) (Lev, 1992).

La stratégie de communication est définie par les responsables de la diffusion (les personnes investies de la direction globale de l'entreprise et les directeurs des services comptables et financiers) et par le conseil d'administration en fonction de leur interprétation des pressions externes et de l'ampleur des coûts associés. Cette interprétation dépend :

- de la personnalité des responsables de la diffusion qui se manifeste dans leur vision de la diffusion qui peut être soit conservatrice où prévaut le «ritualisme», soit dynamique ce qui correspond à une approche "opportuniste» de la diffusion (Gibbins et al., 1992; Lacombe-Saboly, 1994). Cette vision est orientée par les valeurs culturelles personnelles des dirigeants et par les valeurs comptables dominantes dans l'entreprise ainsi que par leurs attaches académiques ou leur intolérance à l'incertitude ;

- des caractéristiques du conseil d'administration puisque les membres qui le composent et leur personnalité influencent les choix stratégiques de communication (Leftwich et al., 1981). Or, lorsque, comme en France, les membres du conseil sont aussi les responsables de l'entreprise, l'influence de leur vision de la diffusion s'en trouve renforcée.

\subsubsection{Les coûts indirects pris en considération.}

L'objet de la stratégie de communication est de réduire les coûts indirects que peut supporter l'entreprise. Chacun de ces coûts renvoie à des facteurs spécifiques interprétés et pris en compte par les responsables de la diffusion et le conseil d'administration. La littérature considère en particulier : 
- les coûts d'agence (Jensen et Meckling, 1976). Ces coûts évoluent selon l'actionnariat, dont la nature (investisseurs institutionnels, internationaux ou l'Etat) et la diffusion modifient la relation d'agence, ou selon la nature des actifs d'exploitation. L'endettement peut alors être un moyen d'encadrer les agents mais il en découle un coût d'agence de la dette (Jacquillat et Levasseur, 1981) ;

- le coût du capital (Elliot et Jacobson, 1994) qui incite l'entreprise à prendre en considération le statut de cotation domestique ou multiple de l'entreprise (lié souvent à l'internationalisation). L'influence du statut de cotation varie selon les caractéristiques des marchés financiers de cotation. La gestion du coût du capital amène l'entreprise à tenir compte des performances réalisées et des décisions de refinancement ;

- les coûts politiques, sociétaux, médiatiques qui découlent de la visibilité de l'entreprise (Watts et Zimmerman, 1986 ; Brown et Deegan, 1998). La visibilité est accrue par la taille de l'entreprise, ses performances ou son appartenance sectorielle, ses décisions de refinancement ou de restructuration, son actionnariat (Etat ou groupe multinationaux) ;

- les coûts de propriété (Verrechia, 1983) qui varient selon les caractéristiques du secteur d'activité (taux de croissance ou importance des barrières à l'entrée), les performances réalisées peuvent alors accroître ces coûts. Néanmoins, les coûts de propriété ne peuvent être totalement évités en raison du mimétisme sectoriel qui s'impose à l'entreprise.

\subsection{L'étape de la production de l'information comptable.}

L'information comptable à communiquer définie par la base schématique est contrainte par la phase de production. L'information diffusée est l'intersection entre la demande externe, ce que veut diffuser l'entreprise et ce qu'imposent les structures internes. Les contraintes des structures internes sont matérialisées par l'organisation comptable (Gibbins et al., 1992).

Les mécanismes théoriques de l'influence de l'organisation comptable reposent sur le modèle de prise de décision, centralisé ou décentralisé, prévalant dans le service comptable. En fonction de ce modèle, l'influence du dirigeant de l'entreprise et de sa vision de la diffusion s'en trouvera plus ou moins renforcée. D'autre part, les décisions de présentation de l'information diffusée sont souvent collectives. En fonction de ce processus collectif, l'information diffusée est le résultat d'un consensus ou d'un compromis (Lacombe-Saboly, 1994).

L'information comptable produite et diffusée dépend des capacités des systèmes d'information de l'entreprise non seulement en terme de quantité mais aussi en terme d'évolution et d'aptitude à prendre en compte les demandes formulées par les parties externes à l'entreprise ainsi que du et le rôle des consultants. La taille de l'entreprise et la position du service au sein de l'entreprise (son implication dans les décisions de production, de refinancement et de gestion courante, sa capacité à réduire l'incertitude, l'existence de réseau de substitution) modifie l'importance accordée à la diffusion.

Plusieurs facteurs affectent l'organisation comptable. Ce sont la régulation comptable nationale et internationale qui s'imposent à l'entreprise par le biais des cabinets d'audit ou dès lors qu'elle accède aux marchés financiers nationaux et internationaux. C'est aussi le comité d'audit qui travaille pour le compte du conseil d'administration et qui a pour mission de d'accroître la qualité de l'information 
communiquée (Thiéry-Dubuisson, 1998). Toutefois, son influence dépend de l'implication des responsables de la diffusion.

\subsection{Les étapes de la communication externe et du retour sur communication.}

L'information à diffuser aux tiers fait l'objet d'une certification par le cabinet d'audit et d'une approbation par l'assemblée des actionnaires. Ces deux derniers facteurs affectent le processus de communication parce qu'ils ont la capacité de faire modifier le contenu et la production de l'information externe. En particulier la fonction d'audit est un moyen privilégié par l'entreprise pour crédibiliser son information externe ; cette nécessité sera d'autant plus grande que l'entreprise est de grande taille ou est cotée sur des marchés étrangers (Nobes et Parker, 1995). L'influence du cabinet d'audit est liée à sa réputation et la nécessité de la préserver en échappant aux pressions des responsables de la diffusion (Watts et Zimmerman, 1986).

Une fois l'information diffusée, le retour sur la communication boucle le processus de communication et concourt à le faire évoluer par l'apprentissage. Cette phase de post évaluation permet de comparer les résultats obtenus par l'entreprise par rapport à ceux attendus (les objectifs poursuivis). La diffusion externe doit offrir une certaine stabilité dans le temps tout en évoluant pour refléter les modifications de l'environnement et les transformations de l'entreprise. Or, les structures de l'organisation et les processus comptables favorisent souvent l'inertie (Gibbins et al., 1990). L'apprentissage contribue à faire évoluer la diffusion par l'intégration des résultats antérieurs (récompenses ou pénalités). Il est donc possible de définir deux aspects de l'apprentissage postérieur à la diffusion. Un premier aspect est l'étude des réactions de l'environnement dans la période qui suit la diffusion externe de l'entreprise et des concurrents (Holland et Stoner, 1996). Un second aspect consiste en l'appréciation des résultats obtenus afin d'acquérir des connaissances utiles pour l'élaboration de la diffusion externe dans le futur. Il faut pour cela que l'entreprise considère les données observées comme non aléatoires et pertinentes pour sa base schématique de diffusion. Si les réactions de l'environnement ne sont pas significatives, l'apprentissage est faible ou nul et renforce les structures et les processus en place. A ce niveau, la visibilité médiatique de l'entreprise (à travers les récompenses attribuées par la presse financière (Gibbins et al., 1989)), le comité de responsabilité sociétale (Cowen et al., 1987) ou le comité d'entreprise (Capron, 1998) émettent des signaux pouvant être intégrés dans la base schématique.

\section{Proposition d'une modélisation du processus de communication.}

La littérature ne pousse pas plus avant la compréhension du processus de communication d'informations externes. A partir de la revue que nous venons d'exposer, nous proposons donc une analyse de ce processus qui aboutit à sa modélisation théorique. Cette modélisation répond à trois objectifs : organiser les faits observés et théorisés par la littérature en structurant et en établissant des relations pour constituer une représentation explicative du phénomène, proposer de nouveaux faits, de nouvelles relations et de nouvelles explications et, enfin, servir de base à l'exploration empirique. Nous exposons dans cette partie notre représentation explicative du processus de communication qui tient non seulement dans une qualification des relations 
identifiables entre les facteurs au cours de chaque étape, qui peuvent être directes ou indirectes (en impliquant un ou plusieurs facteurs en chaîne), mais aussi dans l'adoption d'une schématisation circulaire.

\subsection{Mise en relation des facteurs affectant le processus de communication d'informations comptables.}

La dénomination de chaque facteur explicatif puis le positionnement des facteurs les uns par rapport aux autres et par rapport à chacune des étapes du processus permet de dresser le tableau I. Ce tableau distingue, pour chaque étape du processus de communication, plusieurs catégories de facteurs explicatifs. En premier lieu, il est possible d'identifier des facteurs pivots sur lesquels repose chacune des étapes et qui vont encadrer la diffusion. Ces facteurs peuvent être qualifiés de facteurs " clés ». Ils assimilent l'influence des facteurs "justificatifs ». Ces facteurs sont ainsi dénommés car ils justifient les éléments distinctifs du cadre fixé à la diffusion ou, plus généralement, à l'information comptable. Une dernière catégorie est constituée des facteurs d'« environnement». Ces facteurs ont la capacité de modifier l'influence exercée par les facteurs «justificatifs» ou les facteurs «clés ». Ces facteurs peuvent s'enchaîner, le tableau I distingue donc plusieurs niveaux.

Tableau I : Classification des facteurs explicatifs.

\begin{tabular}{|c|c|c|c|c|}
\hline \multirow{2}{*}{$\begin{array}{l}\text { Etape du processus de } \\
\text { communication }\end{array}$} & \multirow{2}{*}{ Facteurs clés de l'étape } & \multirow{2}{*}{ Facteurs justificatifs } & \multicolumn{2}{|c|}{ Facteurs d'environnement } \\
\hline & & & $1^{\text {er }}$ niveau & $2^{\text {ème }}$ niveau \\
\hline $\begin{array}{l}\text { Elaboration de la stratégie } \\
\text { de communication }\end{array}$ & $\begin{array}{l}\text { Conseil d'administration } \\
\text { Responsables de la diffusion }\end{array}$ & $\begin{array}{l}\text { Comité d'audit } \\
\text { Responsables de la diffusion } \\
\text { Cotation } \\
\text { Actionnariat } \\
\text { Visibilité } \\
\text { Secteur d'activité } \\
\text { Décisions de refinancement } \\
\text { Décisions de restructuration } \\
\text { Performances } \\
\text { Structures financières } \\
\text { Actifs d'exploitation } \\
\text { Normalisation comptable }\end{array}$ & $\begin{array}{l}\text { Cabinet d'audit } \\
\text { Attaches académiques } \\
\text { Culture } \\
\text { Aversion au risque } \\
\text { Marché financier } \\
\text { Taille } \\
\text { Taille } \\
\text { Cotation } \\
\text { Taille } \\
\text { Actionnariat } \\
\text { Internationalisation } \\
\text { Performances } \\
\text { Secteur d'activité } \\
\text { Performances } \\
\text { Environnement économique } \\
\text { Organisation comptable } \\
\text { Actionnariat } \\
\text { Visibilité } \\
\text { Secteur d'activité } \\
\text { Visibilité }\end{array}$ & $\begin{array}{l}\text { Normalisation comptable } \\
\text { Environnement } \\
\text { économique } \\
\text { Internationalisation }\end{array}$ \\
\hline $\begin{array}{l}\text { La production de } \\
\text { l'information comptable }\end{array}$ & Organisation comptable & $\begin{array}{l}\text { Taille } \\
\text { Normalisation comptable } \\
\text { Cotation } \\
\text { Comité d'audit }\end{array}$ & $\begin{array}{l}\text { Culture } \\
\text { Système économique } \\
\text { Internationalisation } \\
\text { Marché financier } \\
\text { Responsables de la diffusion }\end{array}$ & Normalisation comptable \\
\hline Diffusion de l'information & $\begin{array}{l}\text { Cabinet d'audit } \\
\text { Assemblée des actionnaires }\end{array}$ & & & \\
\hline
\end{tabular}




\subsection{Typologie des relations recensées.}

Ce regroupement des facteurs invite donc à la classification des interactions qui s'établissent entre eux et avec les étapes du processus de communication. Nous proposons à la fois une définition de la nature de relations qui découlent de cette analyse a priori du processus, mais aussi du sens et de la forme qu'elles sont susceptibles d'adopter. Cette entreprise présente l'intérêt de mieux appréhender le processus complexe de la communication externe au sein d'une entreprise et de le formaliser, ce qui approfondit la problématique commune de l'offre d'information comptable. Pour ce faire nous calquons notre démarche sur celle développée par Wanty et Federwich (1970) pour modéliser une entreprise dans l'optique de la gérer et de prendre les décisions adéquates.

Nous proposons de distinguer quatre grandes natures de relations : des relations de déterminisme, des relations de conditions, des relations de contingence et des relations de médiation. Dans une relation de déterminisme, un facteur traduit une donnée ou une situation qui détermine les caractéristiques des choix effectués. Cette nature de relation implique donc une décision issue de la perception et de l'interprétation de stimuli. Une relation de condition traduit l'existence d'une contrainte ou d'un paramètre généré par un facteur qui est pris en compte et conditionne une action. Une relation de contingence intéresse deux facteurs dont l'un traduit une donnée interne ou d'environnement externe modifiant les caractéristiques de l'autre facteur. Il s'agit ici d'une relation traduisant un phénomène technique ou économique. Enfin, une relation de médiation lie deux facteurs dont l'un permet l'action du second.

L'étude du sens des relations invite à préciser si un facteur exerce une influence positive ou négative à chaque niveau où il intervient. La revue de la littérature théorique propose des sens en majorité positifs. Toutefois, nous avons vu lors de la revue de la littérature que les sens des relations théoriques ne sont pas systématiquement confirmés par les résultats des études empiriques. De plus, le sens d'une relation ne doit pas être dissocié de sa forme puisque certains facteurs agissent en interaction. Ainsi, Wanty et Federwisch (1970) distinguent-ils l'interaction multiplicative, où les effets des deux facteurs se renforcent l'une l'autre de manière plus qu'additive, de l'interaction d'opposition où les effets des deux facteurs se combattent l'un l'autre. La forme de nos relations dépend de leurs natures et semble n'être intéressante à approfondir que dans le cadre des relations de conditions. En effet, ces relations, en présentant un facteur amont comme le paramètre d'une relation, sont susceptibles d'adopter l'une de ces deux formes, multiplicative ou d'opposition.

\subsection{Schématisation du processus de communication.}

En reprenant les quatre étapes du processus identifiées par la littérature et notre classification des relations identifiables selon leur nature, leur sens et leur forme, la figure I présente notre modélisation de ce processus au sein d'une organisation qui met en exergue le rôle central des acteurs internes.

Cette modélisation s'appuie sur un schéma de type circulaire puisqu'elle insère l'étape de l'apprentissage. Cette schématisation renforce également le fait que nombre des facteurs impliqués lors de l'élaboration de la stratégie se trouvent modifiés (renforcés ou atténués) par le retour sur communication. La qualité de l'information diffusée n'est 
donc plus que le symptôme d'un mécanisme complexe que notre modélisation tente d'éclairer.

Figure I : Modélisation du processus de communication.

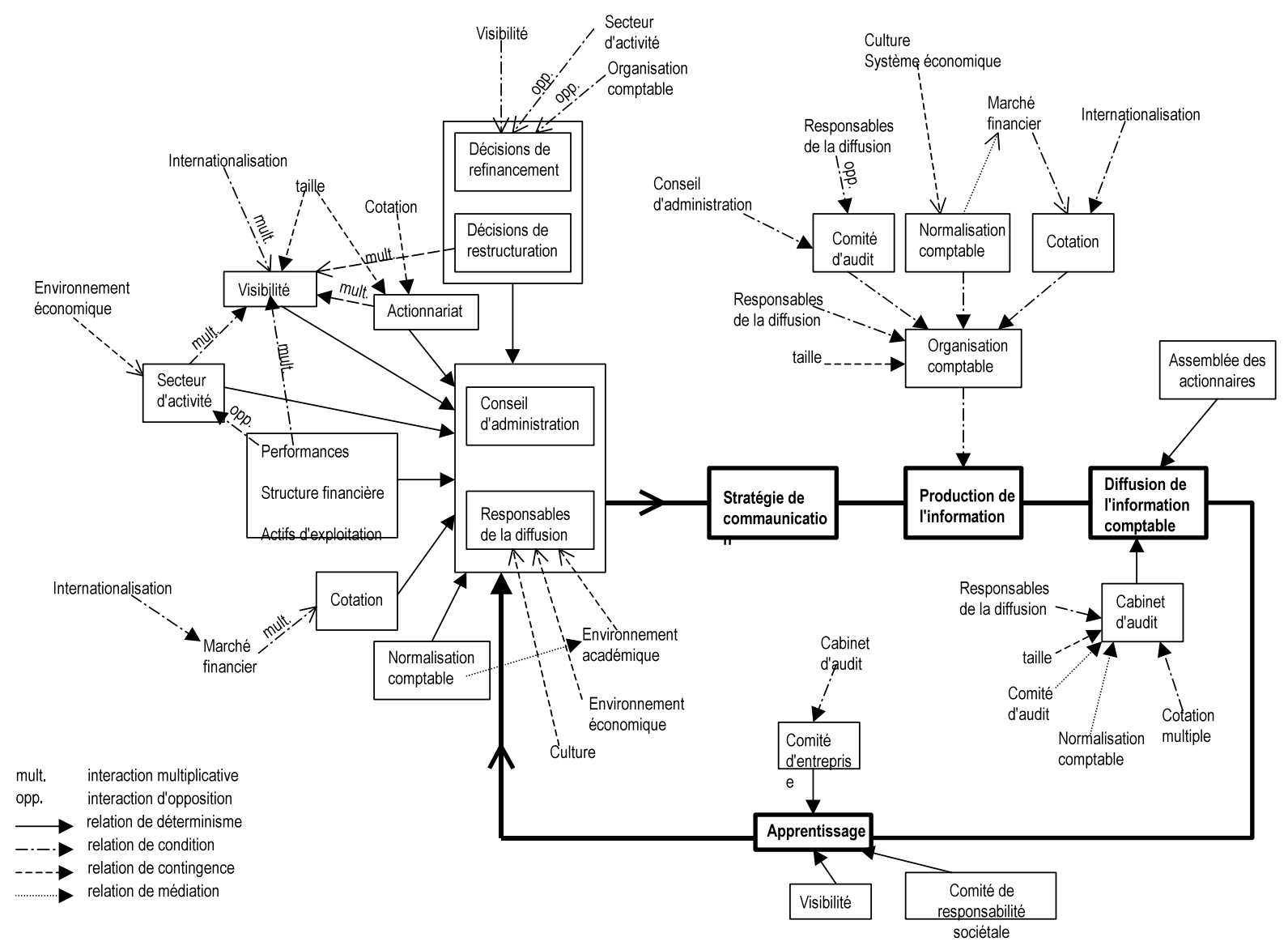

\section{Conclusions et perspectives.}

En réalisant une analyse et une synthèse de la littérature théorique et empirique sur la diffusion, la modélisation du processus de communication présentée dans cet article ouvre de nouvelles perspectives autant pour la recherche quantitative que pour la recherche qualitative. Ceci parce qu'il met en évidence la nécessité de la recherche de relations causales stables et celle des prendre en compte les problèmes réels auxquels sont confrontées les organisations dans la diffusion de l'information externe (Cohen, 1997). Est ainsi mise valeur l'importance de renouveler les méthodologies traditionnelles afin de pointer les orientations susceptibles d'enrichir les connaissances sur la diffusion d'information.

Commençons par évoquer les voies de renouvellement des outils suggérées par cette analyse du processus de communication pour la recherche quantitative. Notre classification aborde différemment l'influence de certains facteurs, traditionnellement présentée comme déterminante: tel est le cas de la taille d'une organisation dont l'influence a été vérifiée par de nombreux auteurs. Toutefois, les arguments mis en avant pour justifier cette influence impliquent tout à la fois les besoins de l'entreprise induits 
par son développement que sa visibilité par exemple. Ainsi, les vérifications empiriques tendent à en faire un facteur causal direct sur les décisions prises en matière de diffusion alors qu'il apparaît comme un facteur contingent et c'est bien le sens des critiques qu'adressent certains auteurs comme Owasu-Ansah (1997): peut-on considérer que la taille pèse sur les décisions de diffusion pour elle-même ou pour des raisons techniques?

Le positionnement des facteurs explicatifs par rapport à l'offre d'information comptable et la qualification des relations potentielles tels que nous le proposons permettent dans ce contexte de mettre en exergue une contrainte importante pour la recherche quantitative: celle de prendre en considération le niveau d'influence des facteurs étudiés. Cette contrainte possède deux implications. D’une part, il devient nécessaire de contrôler l'influence des facteurs d'environnement endogènes, les chaînes de relations ne peuvent pas être ignorées dans l'investigation empirique. D'autre part, plus globalement, il serait intéressant d'adopter une démarche de validation quantitative intégrant le processus en tant que modèle et non plus de manière statique en considérant indépendamment chaque relation, ce qui conduit à une simplification de la réalité. Cheng (1992), en recourant au modèle statistique LISREL développée par K. Joreskog, initie une telle approche puisque cette démarche de validation permet à la fois de tester chaque facteur à travers la variable opérationnelle la plus pertinente, mais aussi d'évaluer l'adéquation globale du modèle avec la réalité, c'est-à-dire la validité du modèle. Cette démarche offre l'opportunité d'apporter une dimension plus dynamique à la recherche quantitative des déterminants de la diffusion.

Mais plus encore, notre analyse du processus de communication met l'accent sur l'intérêt des méthodologies qualitatives qui offrent des pistes de recherche nombreuses et enrichissantes axées sur la compréhension des actions des individus et des facteurs d'influence (Chevalier-Kuszla, 2000). Notre modélisation du processus de communication externe constitue une cartographie du processus de communication, donc un schéma interprétatif d'une entreprise mettant en évidence la finalisation, l'organisation et l'animation impliqués par la diffusion d'information (Cohen, 1997). Elle invite ainsi à renouveler les questionnements afin d'approfondir chaque étape du processus selon plusieurs directions.

L'étape de la formalisation de la stratégie de communication est une étape encore mal précisée et sa compréhension implique, à partir de notre modèle, d'envisager de quelle manière les différents facteurs sont intégrés, le degré de discrétion possédé par rapport à chacun d'entre eux. Ce qui revient à confirmer, infirmer ou raffiner la classification des relations du modèle. Cette voie de recherche permettrait d'approfondir le processus de prise de décision, domaine abordé récemment par Holland et Stoner (1996) ou Chantiri (2000), et qui offre l'intérêt d'étudier le degré de formalisation et de complexité de l'étape au sein d'une organisation. Elle permettrait également d'éclairer l'incidence de l'apprentissage sur la stratégie, puisque celle-ci découle d'un jugement ex ante et a posteriori sur les enjeux de la diffusion. En effet, la manière dont le retour sur informations peut affecter les facteurs (réactions de l'environnement), les acteurs et les étapes du processus (appréciation des résultats) n'est encore qu'évoquée dans la littérature. Notre modèle souligne la faiblesse de la connaissance concernant cette étape. Il s'agit donc dans cette perspective d'approfondir le modèle et les relations potentielles entre deux étapes distinctes du processus. De ce point de vue, les relations entre les étapes de la stratégie et de la production de l'information comptable sont peu développées dans la littérature. Gibbins et al. (1992) qui ont développé la notion 
d'organisation comptable se sont concentrés sur la caractérisation de cette étape. L'aspect des interrelations entre la stratégie et la production de l'information est par contre encore lacunaire. Ceci invite à approfondir le processus de prise de décision et de sa mise en œuvre par exemple selon l'approche de Holland et Stoner (1996) qui étudient une situation spécifique de modification des obligations de diffusion.

Enfin, une dernière piste d'investigation ouverte par l'analyse du processus de communication porte sur son positionnement par rapport aux autres formes de communication d'information comptable, et en particulier la communication privée d'information très importante dans l'entreprise (Sranon-Boiteau, 1998). En fait, la modélisation proposée sépare la communication externe des autres formes de communication alors qu'elle est définie en fonction de la communication privée par exemple (Holland, 1998).

En abordant le fonctionnement d'une organisation du point de vue de la communication d'information comptable externe et en le formalisant pour synthétiser et prolonger la réflexion théorique, les limites de l'investigation quantitative commune mais aussi les enjeux de la recherche sur ce thème sont plus manifestes. Il en ressort que les perspectives de développement ouvertes sont larges à condition de dépasser les problématiques positivistes pures.

\section{Bibliographie.}

Brown R. et Deegan C. (1998), « The public disclosure of environmental performance information », Accounting and Business Research, vol 29, $\mathrm{n}^{\circ} 1$, pp. 21-41.

Capron M. (1998), «Comptabilité et gestion dans le dialogue social : l'expérience de cinquante années de comités d'entreprise en France », $4{ }^{\text {ème }}$ journées d'histoire de la comptabilité et du Management.

Chantiri R. (2000), Contribution à l'analyse des processus d'élaboration des normes comptables : une étude comparée des processus français et britanniques, thèse de sciences de gestion, Université de Paris-Dauphine.

Cheng R. (1992), «An empirical analysis of theories on factors influencing state governement accounting disclosure », Journal of Accounting and Public Policy, vol 11, n¹, pp. 1-42.

Chevalier-Kuszla C. (2000), «Ethnologie et recherche comptable», dans Encyclopédie de Comptabilité, Contrôle de Gestion et Audit de B. Colasse, Economica.

Cohen E. (1997), «Epistémologie de la gestion », dans Encyclopédie de gestion de P. Joffre et Y. Simon, tome 1, Economica.

Cowen S., Ferreri L. et Parker L. (1987), «The impact of corporate characteristics on social responsability disclosure : a typology and frequency-based analysis ", Accounting, Organizations and Society, vol 12, n², pp. 111-122.

Crozier M. et Friedberg E. (1977), L'acteur et le système, Editions du Seuil.

Dye R. (1985), «Strategic accounting choice and the effects of alternative financial reporting requirements », Journal of Accounting Research, vol 23, n², pp. 544-574.

Elliott R. et Jacobson P. (1994), « Costs and benefits of business information disclosure », Accounting Horizons, vol 8, n 4 , pp. 80-96.

Gibbins M., Richardson A. et Waterhouse J. (1989), « Si les chiffres pouvaient parler », CA Magazine, octobre, pp. 29-36.

- (1990), «The management of corporate financial disclosure : opportunism, ritualism and processes », Journal of Accounting Research, vol 28, n¹, printemps, pp. 121-143.

- (1992), The management of financial disclosure : theory and perspectives, La Fondation de Recherche de l'Association des Comptables Généraux Licenciés du Canada, monographie de recherche ${ }^{\circ} 20$.

Healy P. et Palepu K. (1993), « The effects of firms' financial disclosure strategies on stock prices », Accounting Horizons, vol 7, $\mathrm{n}^{\circ} 1$, pp. 1-11.

Holland J. (1998), «Private volutary disclosure, financial intermediation and market efficiency », Journal of Business, Finance and Accounting, vol 25, n 1 et 2, pp. 29-68.

Holland J. et Stoner G. (1996), «Dissemination of price-sensitive information and management of voluntary corporate disclosure », Accounting and Business Research, vol 26, n 4, pp. 295-313. 
Hopwood G. (1983), «On trying to study accounting in the contexts in which it operates », Accounting, Organizations and Society, vol. 8, $\mathrm{n}^{\circ} 2 / 3$, pp. 287-305.

Jacquillat B. et Levasseur M. (1984), « Signaux, mandats et gestion financière : une synthèse de la littérature », Finance, vol 5, janvier, pp. 7-83.

Jensen M. et Meckling W. (1976), «Theory of the firm : managerial behaviour, Agency costs and ownership structure », Journal of Financial Economics, vol 3, n²4, pp. 305-360.

Lacombe-Saboly M. (1994), Les déterminants de la qualité des produits comptables des entreprises : le rôle du dirigeant, thèse de science de gestion, Université de Poitiers.

Leftwich R., Watts R. et Zimmerman J. (1981), "Voluntary corporate disclosure : the case of interim reporting », Journal of Accounting Research, ${ }^{\circ} 19$, supplement, pp. 50-77.

Lev B. (1992), « Information disclosure strategy », California Management Review, vol 34, n4, pp. 9-32.

Michaïlesco C. (1999), Une étude empirique des déterminants de la qualité de l'information diffusée par les entreprises françaises au cours de la période 1991-1995 », Comptabilité, Contrôle, Audit, mars, pp. 83-108.

Owusu-Ansah S. (1997), "The determinants of voluntary financial disclosure by Swiss listed companies : a comment », The European Accounting Review, vol. 6, n 3, pp. 487-492.

Sranon-Boiteau C. (1998), De l'utilisation de l'information comptable par les analystes financiers français, thèse de sciences de gestion, Université de Paris-Dauphine.

Thiéry-Dubuisson S. (1998), «La diffusion des comités d'audit aux Etats-Unis et en France: Une étude comparative », dans Performances et comptabilité, Actes du XIX ${ }^{\text {ème }}$ congrès de l'AFC, tome 1, pp. 243-258.

Verrecchia R. (1983), « Discretionary disclosure », Journal of Accounting and Economics, vol 5, n³, décembre, pp. 179-194.

Wanty J. et Federwisch J. (1970), Les modèles globaux d'économie d'entreprise, Dunod.

Watts R.L. et Zimmerman J.L. (1986), Positive accounting theory, Prentice-Hall. 